\title{
A 6.25 MHz BW 8-OSR Fifth-Order Single-Stage Sigma-Delta ADC
}

\author{
Chang-Seob Shin, Min-Ho Yoon, Kang-Il Cho, Young-Ju Kim, Kwang-Soo Kim, Seung-Hoon Lee, Gil-Cho Ahn \\ Dept. of Electronic Engineering \\ Sogang University \\ \#1 Sinsoo-Dong, Mapo-Gu, Seoul, 121-742, Korea \\ gcahn@sogang.ac.kr
}

\begin{abstract}
A switched-capacitor single-stage sigma-delta ADC with a fifth-order modulator is proposed. The proposed sigmadelta ADC employs feed-forward architecture with oversampling ratio (OSR) of 8 . The modulator input signal range is extended beyond the full scale of the quantizer with proper coefficients scaling and internal DAC reference scaling. A 19-level quantizer with data weighted averaging dynamic element matching (DWA DEM) technique is employed to improve the linearity of a multi-bit DAC. The prototype ADC fabricated in a $0.13-\mu \mathrm{m}$ CMOS technology achieves $63.7 \mathrm{~dB}$ SNDR with $1 \mathrm{MHz}$ input signal over $6.25 \mathrm{MHz}$ signal bandwidth while consuming $52.5 \mathrm{~mW}$ with the clock frequency of $100 \mathrm{MHz}$.
\end{abstract}

\section{INTRODUCTION}

The sigma-delta architecture has been chosen for the applications requiring high signal-to-noise ratio (SNR) with limited signal bandwidth due to its oversampling. However, improvements in technologies make it possible to use sigmadelta analog-to-digital converters (ADCs) for wide-band applications such as mobile communication with increased operation speed. Oversampling and noise shaping properties of the sigma-delta modulator relax the requirements of antialiasing filter and amplifiers in the integrators which result in reduced power consumption and complexity of the front-end receiver system.

In the applications where above few megahertz signal bandwidth is required, the available OSR of the sigma-delta modulators are limited by the maximum sampling frequency of the given technology. To compensate for the SNR reduction caused by low OSR, it is necessary to use high order sigmadelta modulator. However, stability issue in high order modulators limits the stable input signal range resulting in peak SNR reduction. Especially, with the aggressive supply scaling in modern deep-submicron technologies, the signal power scales down proportionally, thus, it becomes more difficult to maintain same peak SNR.

To avoid stability problem without the reduction of the input signal range, multi-stage noise shaping (MASH) topologies are frequently used for the implementation of high order sigma-delta modulators [1]. While the MASH structures enable the easy realization of stable high order modulator with adequate input range, these are prone to quantization noise leakage due to mismatch between the analog modulator and the digital noise cancelation filter. Typically, high DC gain operational amplifiers (op-amps) are required in MASH structures to prevent quantization noise leakage. However, in deep-submicron processes, it is difficult to achieve high DC gain because of the low output resistance of transistors. Even though high DC gain of the op-amps can be achieved by using multi-stage amplifiers, it requires more power consumption and area.

In contrast to MASH structures, the DC gain requirement of the op-amps in single-stage high order modulators can be relaxed at the expense of limited stable input signal range [2], [3]. Therefore, in single-stage high order sigma-delta ADCs, stable input signal range needs to be maximized to improve the achievable peak SNR. The difficulty in implementing stable single-stage modulators at low OSR can be somewhat alleviated by using an internal multi-bit quantizer and digitalto-analog converter (DAC). Moreover, the performance of the modulator can be improved through the increased resolution of the quantizer. However, the nonlinearity caused by the multi-bit DAC of the first integrator is directly referred to the input signal of the modulator and degrades the overall performance. To enhance the multi-bit DAC linearity, a dynamic element matching (DEM) technique is typically employed [4].

In this paper, a multi-bit single-stage sigma-delta ADC by using the simple coefficients scaling method to extend the input signal range is presented. This paper is organized as follows. Section II discusses the proposed sigma-delta modulator architecture. Section III describes details of the circuit implementation. In Section IV, measurements results are presented. The overall conclusion is drawn in Section V.

\section{PROPOSED MODULATOR ARCHITECTURE}

The block diagram of the proposed sigma-delta ADC is illustrated in Fig. 1. It consists of a fifth-order single-stage

The chip was fabricated through the MPW of IC Design Education Center (IDEC) supported by the Korea Ministry of Knowledge Economy (MKE). 


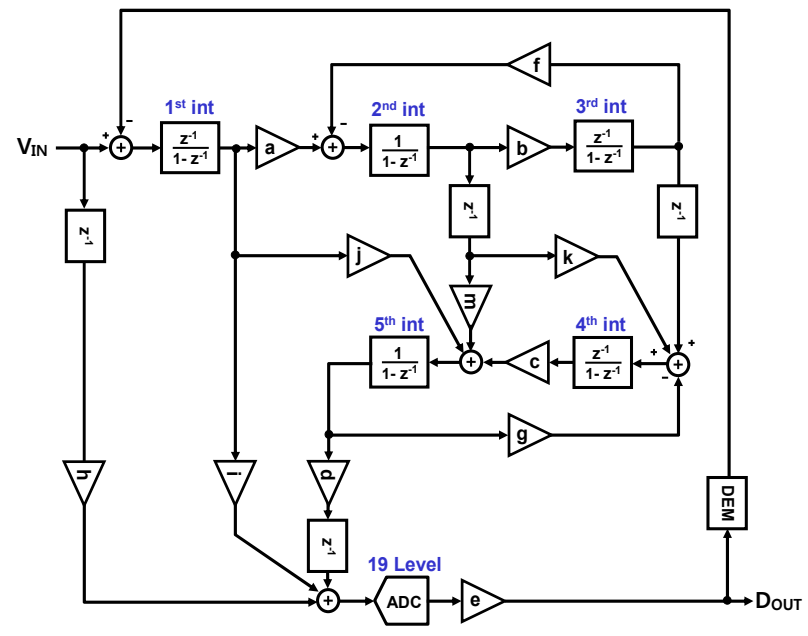

Figure 1. Block diagram of single-stage fifth-order sigma-delta ADC.

sigma-delta modulator containing 19-levels quantizer. Delayed feed-forward path is employed to guarantee the timing margin of the modulator [5]. Allowable input signal range of the modulator is extended by scaling the gain coefficients $e$ and $h$. Two resonators consist of second and third, fourth and fifth integrators are adopted to reduce the inband quantization noise. The distortion introduced by the capacitor mismatches in the multi-bit DAC is attenuated by using the DEM logic.

\section{A. Delayed feed-forward}

The low-distortion feed-forward architecture is employed for the proposed modulator [5]. In conventional feed-forward architecture shown in Fig. 2, the modulator input signal, X, is eliminated by the subtraction of delay free path feedback signal starting from the modulator input to the first integrator DAC input through the quantizer. As a result, the first integrator processes only shaped quantization noise and there is no distortion introduced by the integrator. However, in high speed operation, it is difficult to implement delay free path feedback signal due to the limited timing margin. In the proposed sigma-delta modulator, one clock delay is inserted into the feed-forward path to relax the timing requirement as shown in Fig. 3. With this one clock delay insertion, $n^{\text {th }}$ order shaped input signal appears at the output of the first integrator which is small enough for the target performance of the proposed sigma-delta ADC [6].

\section{B. Input signal range}

Large signal swing at the output of the integrators and the input of the quantizer disturbs the stable operation of singlestage modulators. Even though the feed-forward architecture contributes to lowering the output swing of the integrators, overloading of the quantizer input resulted by the large input signal of the modulator still disturbs the stable operation. To avoid the overloading of the quantizer input and increase stable input signal range, proper coefficients scaling and DAC reference voltage scaling are used. Fig. 3 shows a linearized model of the proposed modulator with loop filter H. Since the

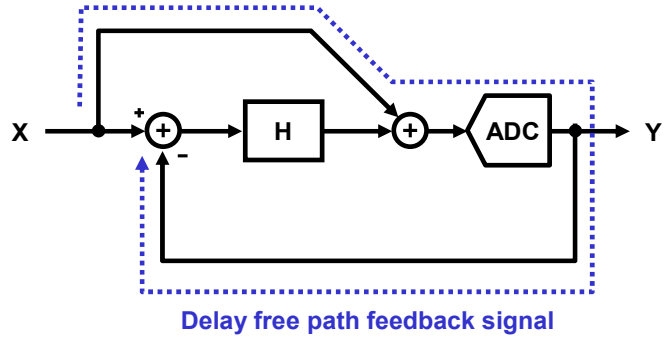

Figure 2. Block diagram of conventional feed-forward sigma-delta modulator.

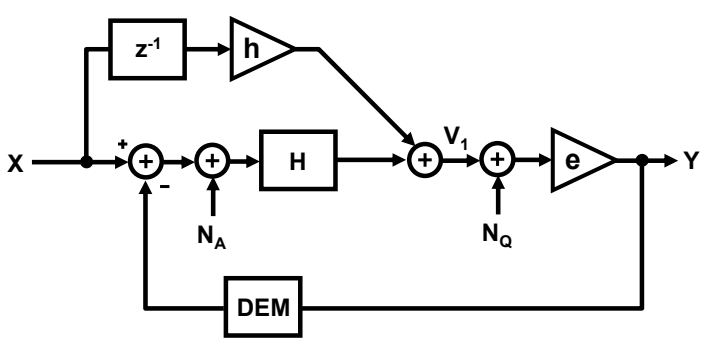

Figure 3. Linearized model of the proposed sigma-delta ADC.

gain from input, $\mathrm{X}$, to the output, $\mathrm{Y}$, is almost unity in the signal bandwidth, node voltage, $\mathrm{V}_{1}$, at the input of the quantizer contains input signal component divided by gain coefficient $e$. To avoid the quantizer overloading, swing range of $\mathrm{V}_{1}$ can be scaled down by increasing the gain coefficient $e$ at the output of the quantizer. The full scale of the DAC is increased as compared with the full scale of the quantizer to cover the amplified digital output by the gain coefficient $e$. The full scale of the quantizer is $0.8 \mathrm{Vp}-\mathrm{p}$, while that of internal DAC is $1.1 \mathrm{Vp}$-p. With this gain coefficient, stable input signal range of the modulator is increased by $1 \mathrm{~dB}$ above the full scale of the quantizer.

\section{Resonators}

In wide-band sigma-delta modulators, requirement of the higher operating clock frequency dramatically increases power dissipation. Moreover, the operating speed is limited by the process technology even with the more power consumption so the allowable OSR is limited. In the proposed modulator, OSR of 8 is selected by considering the process technology and power budget. To improve SQNR with selected low OSR, the proposed modulator employs two notches within the signal band [7]. The closed loop of second, third and forth, fifth integrators make notches respectively.

\section{CIRCUITS IMPLEMENTATION}

\section{A. Amplifiers}

Amplifiers are the most critical circuits which consume most of the power in the sigma-delta modulator. Single-stage folded-cascode amplifiers with the switched capacitor common-mode feedback shown in Fig. 4 are employed to guarantee the low-voltage operation in the proposed sigmadelta modulator. PMOS input differential pair is used to bias 


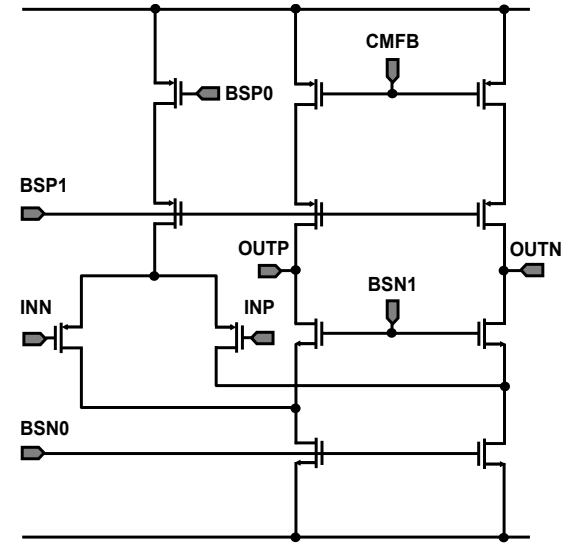

Figure 4. A schematic of the amplifiers.

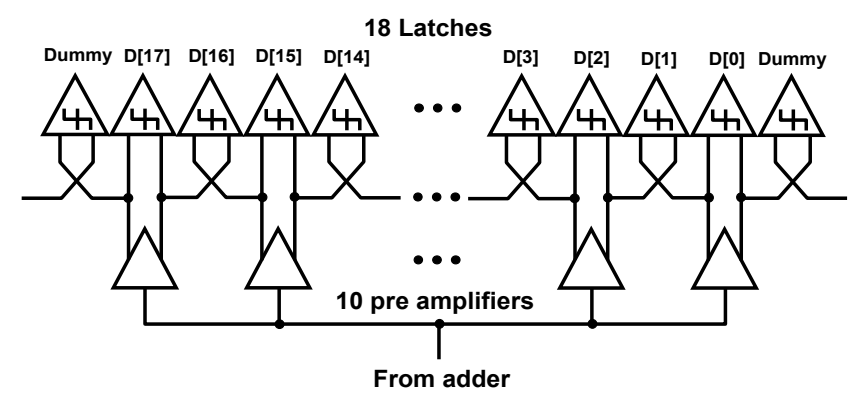

Figure 5. Interpolating quantizer.

the input common-mode voltage of the amplifier near to ground, thus small size NMOS transistors can be used for the switches connected to the input of the amplifiers. Also, the low level input common-mode voltage allows the use of cascoded tail current source for the input differential pair which results in improved common-mode rejection ratio (CMRR) and the accuracy of the bias current. The DC gain requirement of the op-amp is relaxed by using the single stage modulator. A $48 \mathrm{~dB}$ DC gain op-amp is used in the first integrator.

\section{B. Adder}

An active adder using an amplifier is employed for the summing node in front of the quantizer. Although the passive adder can be used without an extra amplifier and power consumption, there is signal attenuation at the input of the comparator which limits the accuracy of the quantizer especially when a multi-bit quantizer is employed in the modulator. To guarantee the reliable operation of the quantizer and reduce the kick back noise from the latches, active adder is used at the expense of the additional power consumption and area.

\section{Interpolating quantizer and DEM}

A 19-level quantizer is used in the proposed modulator. Preamplifiers followed by latches are employed to reduce the input referred offset and kick back from the latches. In order to reduce the number of preamplifiers in the quantizer, interpolating technique is used as illustrated in Fig. 5 [8]. 20

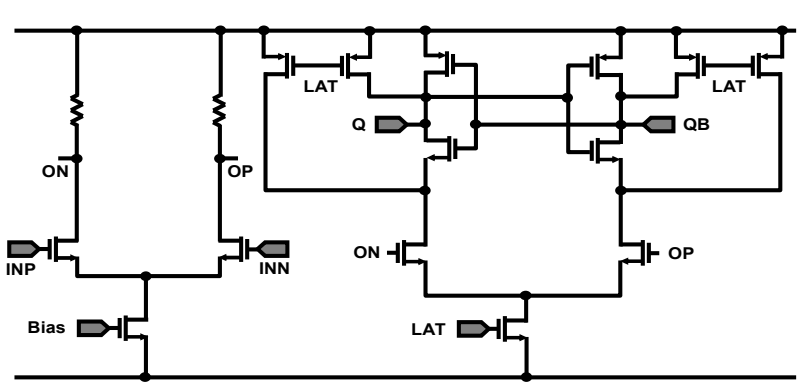

Figure 6. A schematic of the comparator.

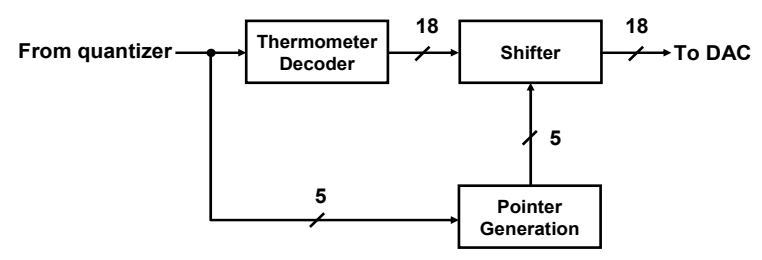

Figure 7. Block diagram of DWA.

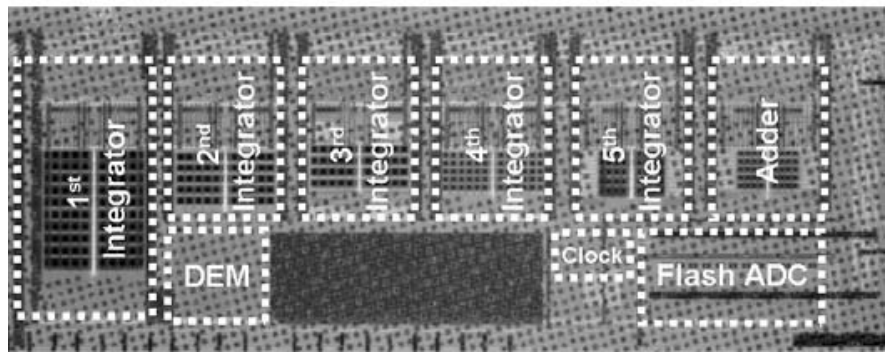

Figure 8. Die photograph.

latches and 12 preamplifiers including dummy pattern are employed in the 19-level quantizer. The schematic of the comparator consists of preamplifier and latch is shown in Fig. 6. When LAT signal is low, the preamplifier amplifies input signal and the latch is in reset mode. When LAT signal goes high, the latch is triggered to regenerate the output signal of the preamplifier. Data weighted averaging (DWA) logic is located in digital feedback path to reduce the mismatch error between DAC capacitors [4]. The block diagram of the DWA is illustrated in Fig 7. The shifter shuffles selected unit DAC capacitors according to the output of the pointer generator. The pointer generator calculates the origin of the selection by using its previous output and the current output of the quantizer.

\section{EXPERIMENTAL RESULTS}

The prototype sigma-delta ADC is implemented in a 0.13$\mu \mathrm{m}$ 6-metal CMOS process. Fig. 8 shows the die photograph of the prototype chip. The active area is $2.02 \mathrm{~mm}^{2}(2.4 \mathrm{~mm} \times$ $0.84 \mathrm{~mm}$ ). Fig. 9 shows the measured power spectrum of the output for a $1 \mathrm{MHz}, 1.1 \mathrm{dBFS}$ sine wave input with $100 \mathrm{MHz}$ clock frequency. The SNDR vs. input amplitude curve is illustrated in Fig. 10. The measured results demonstrate that the modulator can process input beyond the full scale of the 


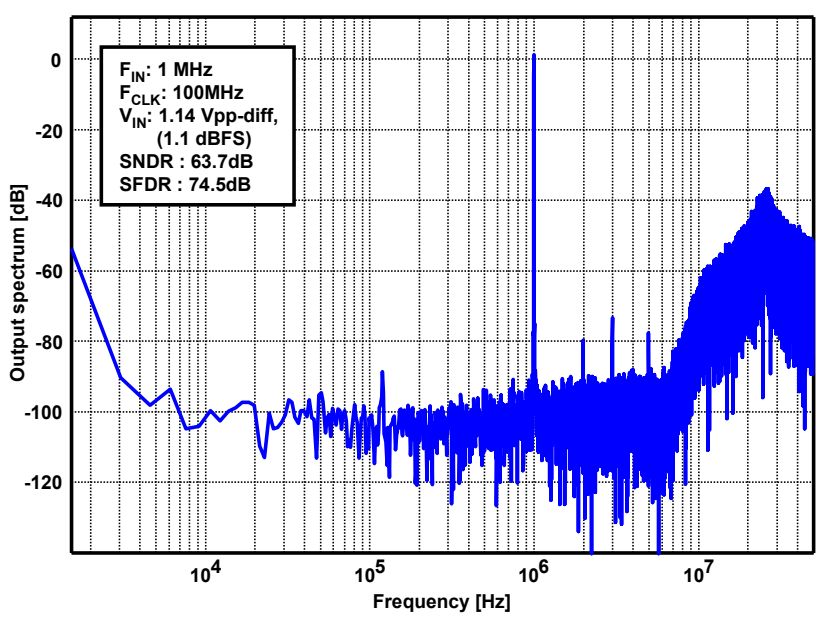

Figure 9. Measured output spectrum.

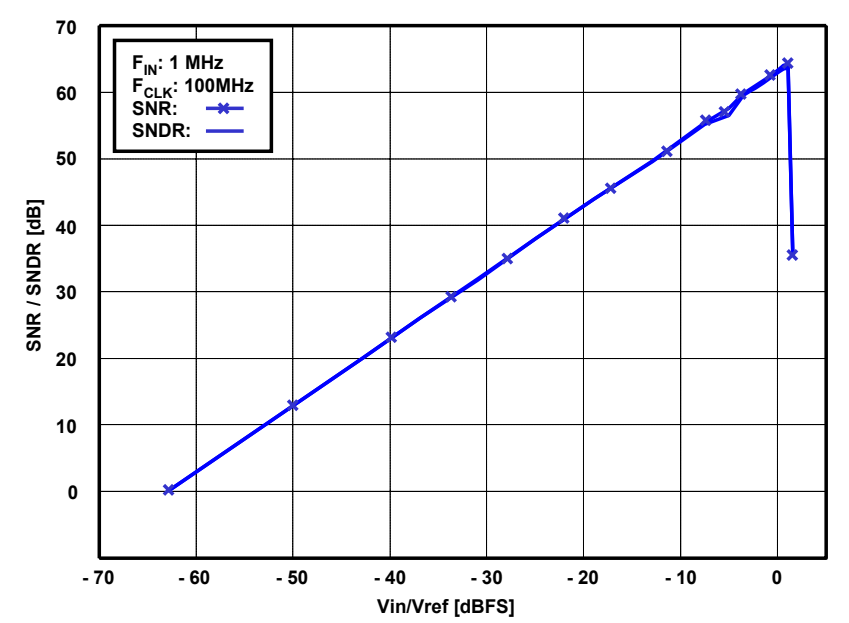

Figure 10. Measured SNDR versus the input level.

quantizer without overloading. The peak SNDR and SNR reach $63.7 \mathrm{~dB}$ and $64.2 \mathrm{~dB}$, respectively. The total power dissipation is $52.2 \mathrm{~mW}$ (analog: $49.2 \mathrm{~mW}$, digital: $3 \mathrm{~mW}$ ) with $1.2 \mathrm{~V}$ supply voltage. The overall measured results are summarized in Table I.

\section{CONCLUSION}

The wide-band single-stage sigma-delta modulator is presented in this paper. The experimental results demonstrate that the proposed sigma-delta $\mathrm{ADC}$ has wide stable input range beyond the full scale of the quantizer by using feedforward architecture and proper coefficients scaling. The measured results of the prototype IC fabricated in a $0.13-\mu \mathrm{m}$ CMOS process verify the operation of the proposed architecture.
TABLE I. MEASURED PERFORMANCE SUMMARY

\begin{tabular}{|c|c|}
\hline Power supply voltage & $1.2 \mathrm{~V}$ \\
\hline Signal bandwidth & $6.25 \mathrm{MHz}$ \\
\hline Clock frequency & $100 \mathrm{MHz}$ \\
\hline Oversampling ratio & 8 \\
\hline Peak SNR & $64.2 \mathrm{~dB}$ \\
\hline Peak SNDR & $63.7 \mathrm{~dB}$ \\
\hline Active die area & $2.02 \mathrm{~mm}^{2}$ \\
\hline Total power dissipation & $49.2 \mathrm{~mW}(\mathrm{~A}), 3 \mathrm{~mW}(\mathrm{D})$ \\
\hline Technology & $0.13 \mu \mathrm{m} \mathrm{CMOS}$ \\
\hline active area & $2.02 \mathrm{~mm}^{2}$ \\
\hline \multicolumn{2}{|c}{ REFERENCES }
\end{tabular}

[1] J. Paramesh, R. Bishop, K. Soumyanath and D. Allstot, "An 11-bit $330 \mathrm{MHz} 8 \mathrm{x}$ OSR $\Sigma \Delta$ Modulator for Next-Generation WLAN," in Dig. Symp. VLSI Circuits, 2006, pp. 166-167.

[2] P. Balmelli and Q. Huang, "A 25-MS/s 14-b 200-mW $\Sigma \Delta$ Modulator in

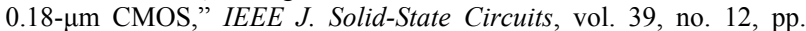
2161-2169, Dec. 2004.

[3] Z. Cao, T. Song and S. Yan, "A $14 \mathrm{~mW} 2.5 \mathrm{MS} / \mathrm{s} 14$ bit $\Sigma \Delta$ Modulator Using Split-Path Pseudo-Differential Amplifiers," IEEE J. Solid-State Circuits, vol. 42, no. 10, pp. 2169-2179, Oct. 2007.

[4] R. T. Baird and T. S. Fiez, "Improved $\Sigma \Delta$ DAC Linearity Using Data Weighted Averaging," Proceedings of the 1995 IEEE International Symposium on Circuits and Systems, vol. 1, pp. 13-16, May 1995.

[5] J. Silva, U. Moon, J. Steensgaard, and G. Temes, "Wideband LowDistortion Delta-sigma ADC topology," Electron. Lett., vol. 37, no. 12, pp. 737-738, Jun. 2001.

[6] H. Park, K. Nam, D. K. Su, K. Vleugels, and B. A. Wooley, "A 0.7-V $100-\mathrm{dB} 870-\mu \mathrm{W}$ Digital Audio $\Sigma \Delta$ Modulator," in Dig. Symp. VLSI Circuits, Jun. 2008, pp. 178-179.

[7] R. Schreier and G. Temes, Underdtanding Delta-Sigma Data Converter. New York: Wiley/IEEE Press, 2004.

[8] C. Lane, "A 10-Bit 60MSPS Flash ADC," Proc. BCTM, pp. 44-47, Sept. 1989. 